\title{
Control of Cell Adhesion using Hydrogel Patterning Techniques for Applications in Traction Force Microscopy
}

\author{
Joel Christian ${ }^{1}$, Johannes W. Blumberg ${ }^{2}$, Dimitri Probst ${ }^{2}$, Cristina Lo Giudice ${ }^{1}$, Sandra Sindt ${ }^{3}$, Christine Selhuber- \\ Unkel $^{3}$, Ulrich S. Schwarz ${ }^{2}$, Elisabetta Ada Cavalcanti-Adam ${ }^{1}$ \\ ${ }^{1}$ Department of Cellular Biophysics, Max Planck Institute for Medical Research ${ }^{2}$ Institute for Theoretical Physics and BioQuant, Heidelberg \\ University ${ }^{3}$ Institute for Molecular Systems Engineering (IMSE), Heidelberg University
}

\section{Corresponding Author}

Elisabetta Ada Cavalcanti-Adam

eacavalcanti@mr.mpg.de

\section{Citation}

Christian, J., Blumberg, J.W., Probst, D., Lo Giudice, C., Sindt, S., SelhuberUnkel, C., Schwarz, U.S., CavalcantiAdam, E.A. Control of Cell Adhesion using Hydrogel Patterning Techniques for Applications in Traction Force Microscopy. J. Vis. Exp. (179), e63121, doi:10.3791/63121 (2022).

\section{Date Published}

January 29, 2022

\section{DOI}

$10.3791 / 63121$

URL

jove.com/video/63121

\section{Abstract}

Traction force microscopy (TFM) is the main method used in mechanobiology to measure cell forces. Commonly this is being used for cells adhering to flat soft substrates that deform under cell traction (2D-TFM). TFM relies on the use of linear elastic materials, such as polydimethylsiloxane (PDMS) or polyacrylamide (PA). For 2D-TFM on PA, the difficulty in achieving high throughput results mainly from the large variability of cell shapes and tractions, calling for standardization. We present a protocol to rapidly and efficiently fabricate micropatterned PA hydrogels for 2D-TFM studies. The micropatterns are first created by maskless photolithography using nearUV light where extracellular matrix proteins bind only to the micropatterned regions, while the rest of the surface remains non-adhesive for cells. The micropatterning of extracellular matrix proteins is due to the presence of active aldehyde groups, resulting in adhesive regions of different shapes to accommodate either single cells or groups of cells. For TFM measurements, we use PA hydrogels of different elasticity by varying the amounts of acrylamide and bis-acrylamide and tracking the displacement of embedded fluorescent beads to reconstruct cell traction fields with regularized Fourier Transform Traction Cytometry (FTTC).

To further achieve precise recording of cell forces, we describe the use of a controlled dose of patterned light to release cell tractions in defined regions for single cells or groups of cells. We call this method local UV illumination traction force microscopy (LUVI-TFM). With enzymatic treatment, all cells are detached from the sample simultaneously, whereas with LUVI-TFM traction forces of cells in different regions of the sample can be recorded in sequence. We demonstrate the applicability of this protocol (i) to study cell traction forces as a function of controlled adhesion to the 
substrate, and (ii) to achieve a greater number of experimental observations from the same sample.

\section{Introduction}

When interacting with their extracellular environment, adherent cells exert forces that are mainly mediated by integrin-based focal adhesions connecting the extracellular matrix (ECM) to the actin cytoskeleton. Focal adhesions are multiprotein assemblies that are centered around the binding of integrins to ECM-proteins like fibronectin and collagen. Integrin clustering and growth of focal adhesions is crucial not only for the establishment of a mechanically stable connection, but also for recruitment of other focal adhesion proteins, including those that activate the RhoA pathway for the regulation of cellular contractility ${ }^{1}$. The RhoA-dependent contractility of the actin cytoskeleton allows cells to spread and migrate on the underlying ECM, and also to sense its stiffness $^{2}$. The distribution of traction forces strongly depends on cells' spread area and shape, both of which rely on matrix properties and therefore impact cytoskeletal organization, eventually forming a closed feedback loop between matrix mechanics and cytoskeletal organization ${ }^{3,4}$.

Surface micropatterning techniques allow a defined control of cell shape by creating micron-sized regions presenting ECM adhesive proteins; according to the size of these regions, single cells, or groups of cells that adhere to the micropattern $^{5}$. ECM proteins can be patterned on glass substrates by different approaches such as microcontact printing, photo-patterning or laser-patterning ${ }^{6}$. The use of UV light $(\lambda=185$ or $375 \mathrm{~nm})$ combined with surface antifouling strategies offers the flexibility for designing different shapes and sizes and immobilization of multiple protein types with a high precision near surface edges ${ }^{7,8}$. The regions coated with protein repellent chemicals such as polyethylene glycol (PEG) are protected with either a chromium photomask or a digital mirror device (DMD)-based maskless lithography system. The patterns in the masks allow the exposure to UV light of regions that will then be patterned with ECM proteins. The patterning of hydrogel surfaces with ECM proteins requires a transfer step to remove proteins from the glass surface and crosslink them to the patterns. Alternatively, patterning on soft materials can be achieved by first coating the photomask with a repellent protein, followed by burning the unmasked regions using deep UV illumination. Since deep UV generates ozone and makes the surface reactive for protein binding, the unmasked regions are coated with ECM proteins and finally the gel is polymerized directly on top of the unmasked regions ${ }^{9,10}$.

The patterned hydrogels can be used to perform TFM, a technique that measures cell forces at the cell-material interface $^{11}$. In 2D-TFM, one uses the flat surface of a thick polymer film, in which marker beads have been embedded to track deformations ${ }^{12,13,14,15}$. In order to extract displacement vectors, it is essential to combine two images, one of the deformed state and one reference image without deformations. The two images are then mapped onto each other with image processing. At high marker density, this is usually done with Particle Image Velocimetry (PIV), which is a well-established method to reconstruct hydrodynamic flow. At low marker density and in 3D-TFM, this is usually done with Particle Tracking Velocimetry (PTV), which includes specific features of the experimental data set. An example of 
a computationally cheaper alternative is optical flow, such as the Kanade-Lucas-Tomasi (KLT) algorithm ${ }^{16}$. In the case of hydrogel substrates, fluorescent beads are usually embedded at high density during the material polymerization, and images are recorded before and after cell release upon enzymatic detachment. Enzymatic detachment of adherent cells, e.g., by trypsinization, leads to simultaneous release of cellular tractions from all cells on the hydrogels, making it difficult to obtain a detailed analysis from a high number of cells.

Here we present a protocol to prepare micropatterned hydrogels to control cell shape and localization and a method to efficiently measure traction forces in sequence using UV to detach individual cells from the substrate. For traction force measurements, we present a technique to produce dual-layered PA hydrogels, where fluorescent beads are embedded only in the top layer, which increases their density and reduces their vertical spread. Combining UV-mediated release of cellular traction forces with micropatterning makes it possible to obtain spatial control over cell detachment (e.g., of single cells without affecting the adhesion of other cells in the region of interest), provided there is a sufficient distance between patterned cells. Cellular traction is then reconstructed using the most efficient and reliable method for 2D-TFM, namely, Fourier Transform Traction Cytometry (FTTC) with regularization ${ }^{17,18}$.

\section{Protocol}

\section{Preparation of methacrylated coverslips}

NOTE: Glass coverslips are methacrylated to covalently link PA hydrogels and therefore prevent their detachment during the incubation with cells. Microscope glass coverslips are used to achieve high image quality.
1. To prepare a $300 \mathrm{~mL}$ solution, take a clean $400 \mathrm{~mL}$ glass beaker and place it inside a fume hood.

2. Add $10 \mathrm{~mL}$ of double distilled water $\left(\mathrm{ddH}_{2} \mathrm{O}\right)$ into the beaker. Add $18.75 \mathrm{~mL}$ of acetic acid ( $\geq 99.8 \%$ pro analysis, p.a.), $18.75 \mathrm{~mL}$ of 3-(Trimethoxysilyl)propyl methacrylate and $252.5 \mathrm{~mL}$ of ethanol ( $\geq 99.8 \%$ p.a.) using a serological pipette. Pour the solution into a crystallizing dish.

3. Clean $24 \mathrm{~mm}$ round glass coverslips with precision wipes. Place the coverslips in a custom-made polytetrafluorethylene rack.

4. Immerse the rack into the solution and incubate for 15 min at room temperature (RT).

5. Take the rack and rinse all coverslips with ethanol $(99.8 \%$ p.a.). Dry the coverslips under air flow.

NOTE: Methacrylated coverslips can be stored for up to 1 month at RT

\section{Micropatterning of extracellular matrix proteins on glass coverslips}

NOTE: Glass coverslips are first coated with a layer of molecules, comprising of protein and cell repellent. This layer is then removed using a photopatterning technique, to allow the subsequent deposition of extracellular matrix proteins in micropatterned regions.

1. Take a $15 \mathrm{~mm}$ round glass coverslip and place it on a Petri dish. Use a diamond pen to mark the upper side of the coverslip. Then, proceed to cleaning via oxygen plasma treatment at $0.4 \mathrm{mbar}$ and $200 \mathrm{~W}$ for $2 \mathrm{~min}$.

2. Pipette $100 \mu \mathrm{L}$ of $0.01 \%$ poly-L-lysine solution on the surface of each coverslip. Incubate for $30 \mathrm{~min}$ at RT. Wash the coverslips with $10 \mathrm{mM}$ 4-(2-hydroxyethyl)-1- 
piperazineethanesulfonic acid (HEPES) pH 8.5. Remove any excess liquid but keep the surface wet.

3. Pipette $100 \mu \mathrm{L}$ of $50 \mathrm{mg} / \mathrm{mL}$ poly(ethylene glycol) methyl ether succinimidyl valeric acid (mPEG-SVA) in $10 \mathrm{mM}$ HEPES pH 8.5 on the surface of each coverslip. Incubate for $1 \mathrm{~h}$ at RT. Rinse the coverslips with $10 \mathrm{mM}$ HEPES $\mathrm{pH} 8.5$ and dry off under air flow.

4. Add $2 \mu \mathrm{L}$ of UV-sensitive photoinitiator (e.g., PLPP-gel) followed by $40 \mu \mathrm{L}$ of ethanol ( $\geq 99.8 \%$ p.a.) onto the surface. To obtain a homogeneous distribution of the gel and ethanol on the surface, gently tilt the Petri dish back and forth. Wait for $5 \mathrm{~min}$ for the solution to polymerize.

5. Place a single coverslip inside a $35 \mathrm{~mm}$ Petri dish with a $20 \mathrm{~mm}$ hole at the bottom. This allows the laser beam coming from underneath to directly reach the glass surface.

6. Turn on the microscope and a light patterning module (working with this device is only permitted after a safety introduction from the laser safety officers). Calibrate the UV-A laser on the 20x air objective. Put the sample with the photoinitiator on the stage. Adjust the focus on the surface of the glass and turn on the focus control. Load and lock the pre-drawn pattern.

1. Prepare the pattern using a graphics software like Inkscape. Ensure that the pattern file does not exceed the DMD dimensions (1824 x 1140 px, which corresponds to $552 \times 325 \mu \mathrm{m}$ on $20 \times$ lens $(0.28 \mu \mathrm{m} /$ $\mathrm{px})$ ).

NOTE: It is recommended to use the DMD size (1824 x 1140 pixels) as the pattern file size, as this will ensure no error during the patterning. For example, do not produce a pattern file with $1830 \mathrm{x}$ 1130 pixels dimension.
2. For the purpose of pattern transfer to polyacrylamide, ensure that the patterning is done only up to $60 \%-70 \%$ of the radius from the center of the glass to the edge.

3. For patterning a single cell, use a diameter of $50-100$ $\mu \mathrm{m}$ with the distance of $100 \mu \mathrm{m}$ between patterns and a diameter of $150-300 \mu \mathrm{m}$ for a cell group. The appropriate pattern size highly depends on the typical cell size, and it has to be sufficiently large to allow cells to adhere.

7. Start the patterning by UV dose of $30 \mathrm{~mJ} / \mathrm{mm}^{2}$. The patterning duration depends on the number of patterns. Making a single pattern takes approximately $1 \mathrm{~s}$.

8. After completing the patterning step, rinse the surface with phosphate-buffered saline (PBS) three times. Incubate the sample with $100 \mu \mathrm{L}$ of $25 \mu \mathrm{g} / \mathrm{mL}$ fibronectin dissolved in 1x PBS and $25 \mu \mathrm{g} / \mathrm{mL}$ fibrinogen Alexa488 conjugate in PBS for $1 \mathrm{~h}$ at RT. For other ECM proteins, find the optimal concentrations completely covering the patterned regions.

9. Rinse the surface with $1 x$ PBS three times. Ensure that the patterned glass is immediately used for glass-PA transfer. Store the patterned glass in PBS at RT during the hydrogel preparation.

\section{Fabrication of patterned polyacrylamide hydrogels}

NOTE: Polyacrylamide hydrogels are prepared, including oxidized N-hydroxyethyl acrylamide (HEA) to present reactive aldehyde groups for the covalent binding of matrix proteins on the surface. Additionally, a dual-layer approach to embed fluorescent beads only on the top layer of the hydrogel is used 
to improve the recording of bead displacement during traction force microscopy experiments.

1. Put the methacrylated glass coverslips into a Petri dish.

2. To prepare fresh oxidized HEA solution, add $9.55 \mathrm{~mL}$ of double-distilled water into a $15 \mathrm{~mL}$ centrifuge tube. Then, add $0.5 \mathrm{~mL}$ of HEA, and $42 \mathrm{mg}$ of sodium(meta)periodate to get $10 \mathrm{~mL}$ of the solution. Continuously stir the solution in the dark for $4 \mathrm{~h}$.

3. Mix acrylamide, bis-acrylamide, and double-distilled water according to Table 1, to get $10 \mathrm{~mL}$ of stock solution hydrogel (do it under the fume hood, monomers are neurotoxic). Degas and close the lid to an airtight seal. NOTE: The stock solution can be kept in aliquots for up to 1 year at $4{ }^{\circ} \mathrm{C}$. Always characterize the Young's modulus of the hydrogel prior to use.

4. Prepare a dual-layered PA hydrogel (do it under the fume hood, monomers are neurotoxic).

1. Start with the bottom layer by gently mixing $99.3 \mu \mathrm{L}$ of stock solution, $0.5 \mu \mathrm{L}$ of $1 \%$ ammonium persulfate (APS), and $0.2 \mu \mathrm{L}$ of tetramethylethylenediamine (TEMED) in a $1.5 \mathrm{~mL}$ tube. Take $10 \mu \mathrm{L}$ from the solution and pipette it dropwise on the center of the methacrylated coverslip.

2. Carefully place a $15 \mathrm{~mm}$ round coverslip on the droplet and wait for $45 \mathrm{~min}$ for polymerization. Gently detach the top coverslip with a scalpel.

3. For the top layer, gently mix $93.3 \mu$ Lof stock solution with $1 \mu \mathrm{L}$ of fresh oxidized HEA solution, $5 \mu \mathrm{L}$ of fluorescent beads (corresponding to three beads per square micron for beads of $200 \mathrm{~nm}$ sized beads), $0.5 \mu \mathrm{L}$ of $1 \%$ APS, and $0.2 \mu \mathrm{L}$ of TEMED in a 1.5 $\mathrm{mL}$ tube. Take $5 \mu \mathrm{L}$ from the solution and pipette it dropwise on the center of the already polymerized bottom layer.

4. Gently place the micropatterned coverslip on the droplet. Wait for $45 \mathrm{~min}$ at RT for the droplet to polymerize. Make sure the patterned side touches the droplet. Gently detach the coverslip with a scalpel.

5. Glue the $24 \mathrm{~mm}$ coverslips to the bottom of custom-drilled 6-well plates using two-component silicone-glue. After 5 min, add PBS to the wells.

6. Characterize the Young's modulus of the polyacrylamide hydrogel samples by atomic force microscopy (AFM).

1. Mount a spherical tip cantilever on the AFM holder.

2. Calibrate each cantilever by acquiring a forcedistance curve (3-4 V setpoint) on a hard substrate (e.g., glass or mica), extrapolate the cantilever sensitivity, retract from the surface, and perform a thermal tune to get their spring constant.

3. Place the calibrated cantilevers over the hydrogel sample and acquire force curves in PBS buffer, using the following parameters: $20-30 \mathrm{nN}$ force setpoint, 5 or $10 \mu \mathrm{m} / \mathrm{s}$ approach and retraction speed, $5 \mu \mathrm{m}$ ramp size.

NOTE: An inverted optical microscope, equipped with an air 40x objective and a green fluorescent protein (GFP) filter was used to visualize and target ECM-micropatterned areas within the PA hydrogel samples.

4. Plot the acquired force versus distance curves with the AFM analysis software.

5. Find the contact point and convert force versus distance curves into force versus indentation curves. 
6. Fit the indentation part of the curve with Hertz model (or a suitable mechanics model in function of the tip geometry), using a Poisson ratio between 0.2 and 0.5 .

\section{Local release of cell traction forces by UV-A laser illumination (LUVI-TFM)}

NOTE: UV-A laser is applied to release traction forces in cells localized in defined regions of the hydrogels. The UV-A laser (i.e., $\lambda=375 \mathrm{~nm}$ solid-state laser, $<15 \mathrm{~mW}$ ) is a class $3 B$ laser. The unshielded laser beam is dangerous to the eyes and often for the skin. Reflected and scattered light and radiation can be dangerous. Moreover, UV radiation may cause skin cancer. Working with devices is only permitted after safety introduction from the laser safety officers.

1. Aspirate the PBS from the wells.

2. Seed $3 \times 10^{6}$ cells per 6 -well plate in growth medium. For fibroblasts, use high glucose Dulbecco's Modified Eagle Medium (DMEM) containing L-glutamine and supplemented with $10 \%$ Foetal Bovine Serum (FBS) and $1 \%$ penicillin/streptomycin. Incubate the cells overnight at $37^{\circ} \mathrm{C} / 5 \% \mathrm{CO}_{2}$. Wash samples to remove floating cells before starting microscopy.

3. Turn on the microscope incubation chamber and gas supply to obtain a temperature of $37{ }^{\circ} \mathrm{C}$ and $5 \% \mathrm{CO}_{2}$ atmosphere.

4. Turn on the microscope and the laser patterning module. If necessary, re-calibrate the UV-A laser on the 20x air objective using the Leonardo software. Place the custommade 6-well plate with the samples on the stage. Adjust focus on the surface of the PA.
5. Setup the illumination pattern and mark the cells of interest. Refocus on the surface of the hydrogel. Acquire the image of the cells in the brightfield channel. Switch to the Cy5 channel (far-red filter, $650 \mathrm{~nm}$ excitation, $670 \mathrm{~nm}$ emission) and take an image of beads in the deformed state.

6. Shift to the laser channel. Turn on the laser for $3 \mathrm{~min}$. In our particular setup, this corresponded to a light dose of $6,000 \mathrm{~mJ} / \mathrm{mm}^{2}$.

7. Switch to the Cy5 channel and acquire an image of the beads in the undeformed state.

NOTE: For experiment using mouse embryonic fibroblasts, wait for 15 min post-exposure to record the reference/undeformed image (See the Representative Results section). It might be different for other type of cells. Repeat steps 4.5-4.7 again for another cell(s) of interest.

8. To measure the elevated oxidative stress after UVA illumination, use the commercially available reagent (CellRox). CellRox is non-fluorescent in the reduced state and, when oxidized by reactive oxygen species, fluoresces with an emission maximum of $\sim 665 \mathrm{~nm}$.

9. According to the provided protocol, add $5 \mu \mathrm{M}$ reagent into the imaging media and incubate for $30 \mathrm{~min}$ at 37 ${ }^{\circ} \mathrm{C} / 5 \% \mathrm{CO}_{2}$. Then, wash the cells $3 x$ with warm $1 x$ PBS and replace the imaging media. Record the Cy5 signal by fluorescence imaging before and after UV-A illumination using a similar light exposure. 


\section{Image processing, particle image velocimetry and calculation of traction forces}

NOTE: Cell traction forces are recorded by analyzing displacement of fluorescent beads and calculated using image analysis tools based on particle image velocimetry.

1. Open fluorescent beads images, before (i.e., deformed) and after laser (i.e., undeformed) using Fiji. Merge two images as one stack (Image | Stacks | Images to Stack).

2. Correct lateral drift between the two images using StackReg plugin (P. Thévenaz, Swiss Federal Institute of Technology Lausanne). Change images to 8 bit (Image | Type | 8 bit) and re-scale to 1024 x 1024 pix (Image | Scale). Save as an Image Sequence (TIFF format) with the reference image always at the beginning.

3. Acquire the displacement vector field using a cross-correlation technique ${ }^{19}$ from the PIV-field as implemented in the OpenPIV project.

1. Ensure that the relaxed (undeformed) image and the deformed image are covered with search windows of size $w_{R}$ and $w_{D}$ in pixels. For each search window, define a cross-correlation function using the following formula:

$C(r, s)=\frac{1}{w_{R}^{2}} \sum_{i=1}^{w_{R}} \sum_{j=1}^{w_{R}}(R(i, j)-\bar{R})(D(i+r, j+s)-\bar{D})$ Here, $R(i, j)$ and $D(i, j)$ describe the intensity fields of the two images truncated to the selected search window and subsequently mirror padded. $\bar{R}$ and $\bar{D}$ describe their mean value. The window sizes are chosen to be $\boldsymbol{w}_{\boldsymbol{R}}=32$ and $\boldsymbol{w}_{\boldsymbol{D}}=32$ and an overlap of $70 \%$ is used between the neighboring search windows.

2. For each search window determine a displacement vector $\vec{u}=(r, s)^{T}$ that optimizes the crosscorrelation function and assign to the center position of the search window. Subpixel accuracy is obtained using a Gaussian fit around the maxima region as described $^{20}$.

3. Find and remove ambiguous displacement vectors. Displacement vectors corresponding to search windows where the ratio between two highest local maxima of the cross-correlation function below a threshold of 1.5 are considered as ambiguous ${ }^{21}$.

4. Discard displacement vectors that fail the normalized median test for a residual threshold of $1.5^{22}$

5. (Optional) Correct the remaining lateral drift by subtracting a fixed vector $\vec{u}_{0}$ from all displacements. This is done because the displacement in a selected area far away from the cell vanishes.

6. Interpolate the resulting displacement vector field to a regular grid with spacing of $4 \mathrm{px}$ of the input images using a cubic polynomial interpolation. Missing datapoints are filled using a smooth bivariate spline extrapolation. A displacement vector in pixels is related to a local deformation by the pixel ratio of the image $(0.33 \mu \mathrm{m} / \mathrm{px}$ for $20 \mathrm{x}$ air lens $)$.

7. (Optional) Mirror pad the image to reduce ringing artifacts in the subsequent analysis.

8. Multiply the deformation field by a Tukey window function to eliminate the edge effect due to the drift correction, with a parameter of $0.2-0.3$. In this 
experiment, the micropatterned area which is in the center of FOV is of interest.

4. Reconstruct the traction using regularized Fourier Transform Traction Cytometry (FTTC) ${ }^{18}$. As regularization we use the simplest reasonable choice, namely, $0^{\text {th }}$ order Tikhonov (L2) regularization ${ }^{23,24,25}$. An optimal regularization parameter $\lambda$ is chosen such that it minimizes the Generalized Cross Validation (GCV) function defined by 26 :

$G C V(\lambda)=\frac{\sum_{i}\left|\left(\mathbf{G} \boldsymbol{\tau}_{\lambda}\right)_{i}-u_{i}\right|^{2}}{\sum_{i} \mid\left(\left.\left(\mathbf{I}-\mathbf{G} \cdot\left(\mathbf{G}^{T} \mathbf{G}-\lambda^{2} \mathbf{I}\right)^{-1} \mathbf{G}\right)_{i i}\right|^{2}\right.}$

Here $\mathrm{T}_{\lambda}$ is a stacked vector containing the $\mathrm{x}$ and $\mathrm{y}$ components of the reconstructed traction field for the given value of $\lambda$ for all Fourier sampling modes and $\mathrm{G}$ is the linear operator mapping traction fields to their corresponding displacement field, as defined for FTTC. The sum is running over vector components. $u_{j}$ are the $\mathrm{x}$ and y components of the displacement field for all Fourier sampling modes. GCV is widely used in the field of illposed inverse problems and is a good alternative to the L-curve criterion often used in TFM. A Lanzcos filter is used to reduce artifacts introduces due to the limited frequency space and the displacement field upsampling. The traction calculation depends on the Young's modulus of the PA (e.g., $11.3 \mathrm{kPa}$ ) and Poisson's ratio (e.g., for $\mathrm{PA}$ in the range between 0.2 and 0.5 depending on crosslinker concentration).

\section{Representative Results}

The PA hydrogels were polymerized on methacrylated glass coverslips, which present reactive groups for the covalent linkage of the PA. In this way, when placing the hydrogels in an aqueous solution, their detachment from the substrate was prevented (Figure 1A-D). To obtain a high density of fiducial markers near the hydrogel surface, we developed the preparation of a dual-layered PA hydrogel. The bottom layer, without any fluorescent beads, was polymerized on the methacrylated coverslip. Subsequently, another layer containing beads was polymerized on top of the bottom layer (Figure 1E-H), replacing the need for sedimentation, which is often used to achieve bead distribution in a single focal plane and increase bead density. To achieve control over cell shape during TFM measurements, we produced micropatterned PA hydrogels via direct transfer of fibronectinpatterned microstructures from a glass coverslip to the prepolymerized PA (Figure 1F-F'). The addition of $1 \%$ nonconventional crosslinker (oxidized HEA) in the top hydrogel layer solution provides aldehyde groups that covalently bind to amine groups of fibronectin.

We prepared PA hydrogels, which are approximately $60 \mu \mathrm{m}$ in thickness and confined fluorescent beads in the upper layer close to the hydrogel surface. This type of hydrogel is a suitable substrate for imaging cellular tractions with inverted microscopes and thick enough (minimum thickness to perform TFM has been reported to be $20-30 \mu \mathrm{m})^{18}$ to prevent any impact of the glass substrate. Localization of the fluorescent beads was imaged by confocal microscopy (Figure 1I). To visualize protein transfer on the hydrogel, we used fluorescently labeled ECM proteins and imaged them by epifluorescence microscopy (Figure 1J). We prepared micropatterned circles of fibronectin with a diameter of 100 $\mu \mathrm{m}$ (left side) and $50 \mu \mathrm{m}$ (right side) to allow adhesion of groups of cells or single cells, as shown in the overlay of bright field images of adherent cells, and fluorescently labeled fiducial beads and fibronectin micropatterns. On a different sample, the adhesion response of single cells to micropatterned fibronectin was validated by indirect 
immunofluorescence microscopy to image the localization of focal adhesion proteins such as paxillin (Figure 1K).

Both ECM protein coating and hydrogel stiffness are crucial parameters for cell adhesion ${ }^{26}$. To characterize the mechanical properties of our PA hydrogels, we performed nanoindentation experiments by $\mathrm{AFM}^{27}$, coupled with an epifluorescence microscope. Hydrogel substrates of three different stiffnesses were prepared and tested with our setup (Figure 2 and Table 1). Sphere-shaped AFM cantilevers were cyclically approached and retracted from unpatterned PA hydrogels and fibrinogen conjugated to Alexa488micropatterned areas, while recording force-distance curves. Subsequently, contact point evaluation and application of Hertz model allowed us to estimate the Young's modulus (E) of each sample. ECM micropatterning did not alter the Young's modulus, which remained comparable to each of the unpatterned PA hydrogels.

To measure traction forces exerted by adherent cells on the substrate, we developed an experimental setup for reference based TFM carried out on a widefield epifluorescence microscope equipped with near UV laser module (UV-A 6000 $\mathrm{mJ} / \mathrm{mm}^{2}$ ) to release cell tractions (Figure 3A). As the laser beam illumination can be spatio-temporally controlled, not only is it possible to selectively expose a single cell or a cell cluster with a high laser dose, but also and more importantly, the disruptive intermediate step of cell removal from the entire sample using a digestive enzyme like trypsin is no longer necessary.Illuminating cells with such a high laser dose induced an elevated oxidative stress leading to cell death (Figure 3B). Cell death yielded the release of tractions from the substrate, as indicated by the bead displacement (illustrated in Figure 3A). We combined the UV-A illumination with a light patterning module to selectively illuminate micronsized regions of the PA hydrogels (Figure $3 \mathrm{~B}-\mathrm{C}$ ). In this way it is possible to release the tractions of micropatterned cell clusters (Figure 3C,F) or single cells (Figure 3B). Importantly, at our time scales the mechanical properties of the ECM patterned hydrogels were not significantly affected by UV exposure (Figure 2C). Increase in oxidative stress is shown in Figure 3D,E. The traction forces were reconstructed by using regularized FTTC with a regularization parameter chosen by Generalized Cross Validation (Figure 3B,F). The release of forces for single cells occurred over a period of $15 \mathrm{~min}$, and the result of the LUVI-TFM is comparable to the trypsin-based TFM (Figure 3G-H). 


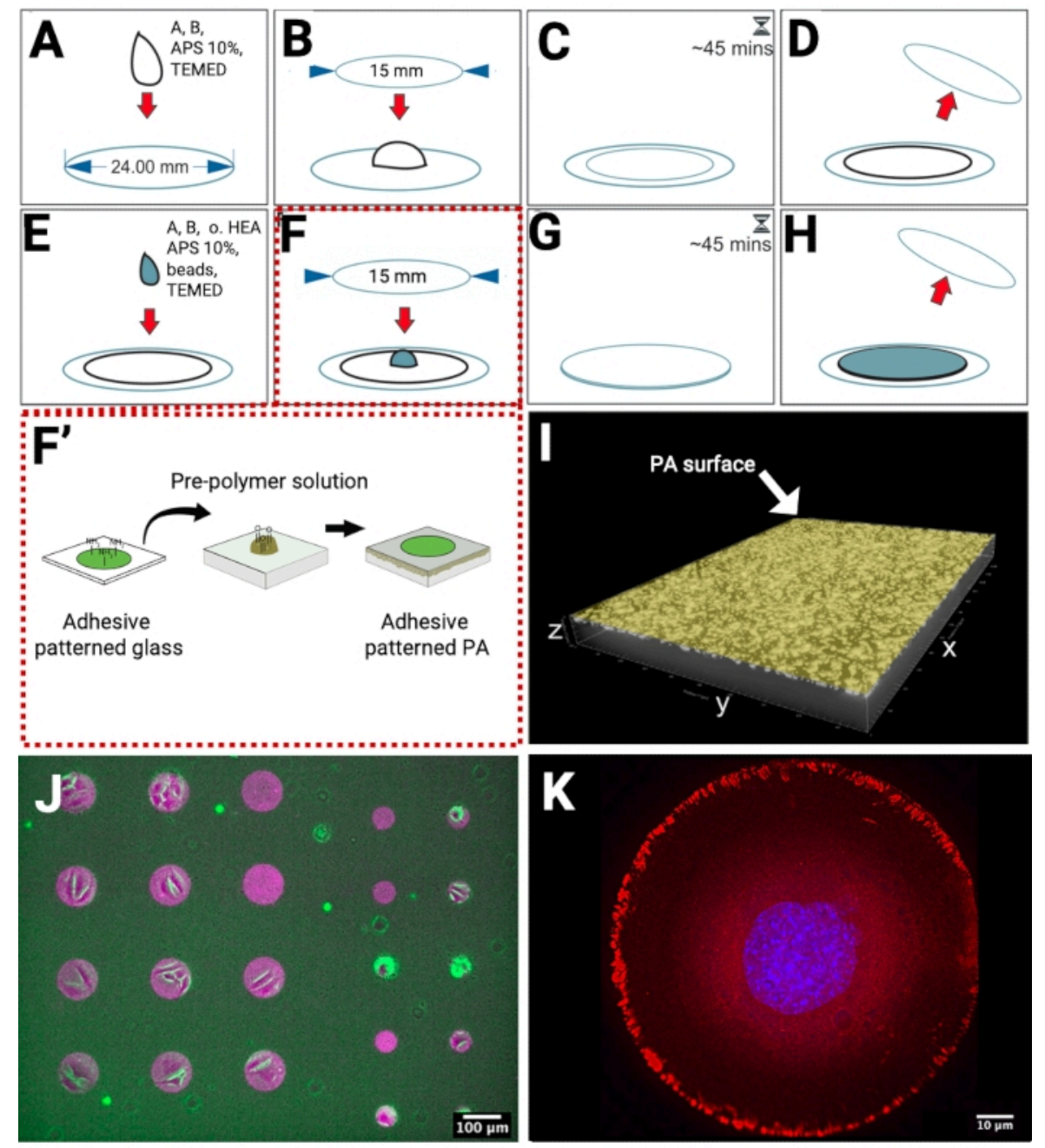

Figure 1: Scheme of patterned fibronectin-substrate preparation for TFM. (A) The solution for the bottom layer is pipetted on a methacrylated surface. (B) A smaller clean coverslip is carefully placed on the droplet. (C) The gelation time is $45 \mathrm{~min}$. (D) The top coverslip is detached. The bottom layer is ready. (E) The solution for the top layer is pipetted on the hydrogel. (F) The patterned coverslip is carefully placed on the droplet. (F') The micropatterning of adhesive proteins on glass is produced by maskless near UV-lithography, and then transferred from glass to PA hydrogel. The free amine groups of adhesive proteins, e.g., fibronectin, bind covalently to aldehyde groups on the PA surface. (G) The gelation time is 45 min. (H) The top coverslip is detached. The patterned PA hydrogel is ready. (I) A 3D representation of a confocal xyz micrograph showing a high density of fiducial beads near the surface. (J) A micrograph of fluorescently labeled fibronectin (magenta) on the surface of PA with embedded fluorescent beads (green), overlayed with a bright field image of cells. (K) Indirect immunofluorescence microscopy imaging of a single cell adhering to a circle-shaped fibronectin micropattern (100 $\mu \mathrm{m})$. Nucleus (blue), paxillin (red). Please click here to view a larger version of this figure. 


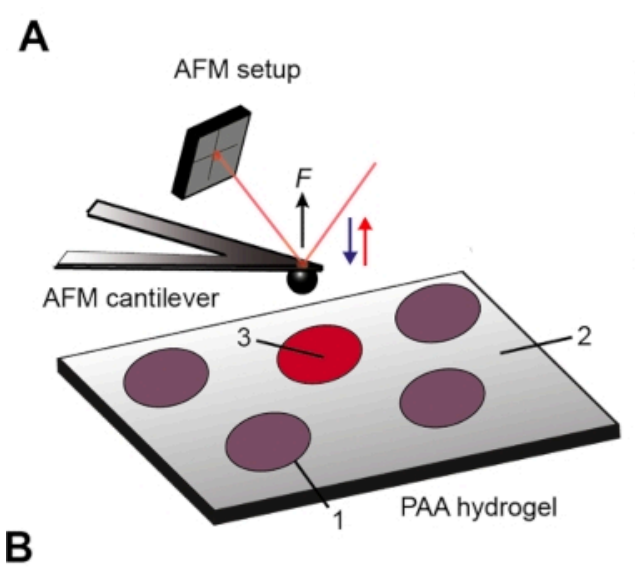

B

1. ECM micropattern

2. No ECM

3. ECM micropattern + UV-A
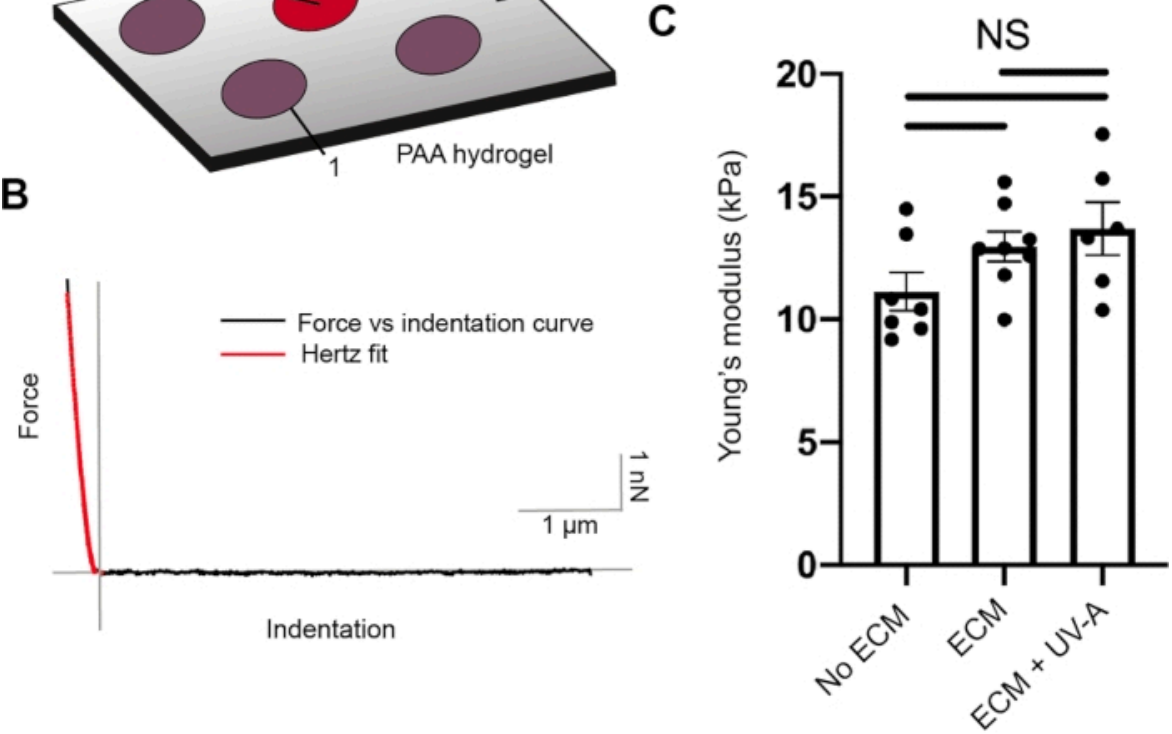

Figure 2: Characterization of sample mechanical properties by AFM. (A) AFM nanoindentation experimental setup. A sphere-shaped cantilever is used to probe ECM micropatterns before or after UV treatment, and the non-patterned regions of dual layered PA (5\% Acrylamide, 0.3\% Bis-acrylamide) areas. (B) Representative force versus indentation curve for ECM micropattern (black). Hertz fit (red) is used to calculate the Young's modulus (E) of the sample. (C) Mechanical measurements for non-patterned dual layered PA areas (no ECM), ECM micropattern and ECM micropattern after UVA exposure. The bars show mean \pm S.E.M. (Brown-Forsythe and Welch ANOVA test). Please click here to view a larger version of this figure. 
A

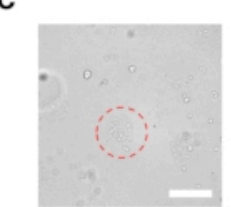

F
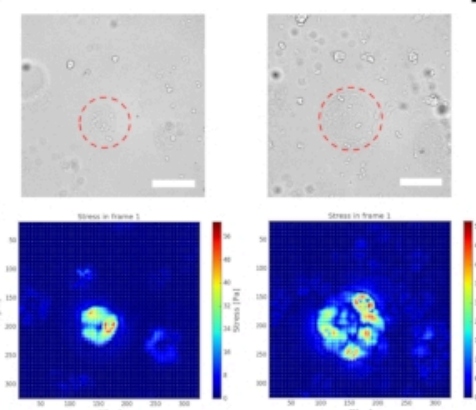

B

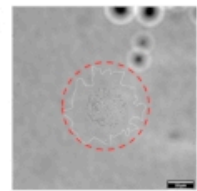

D

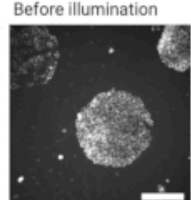

After illumination

E
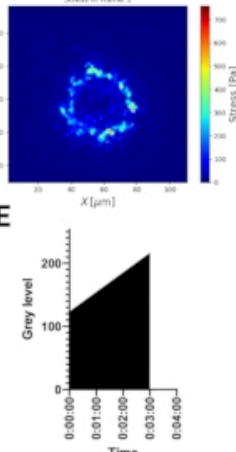

G

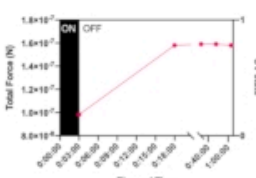

$\mathrm{H}$

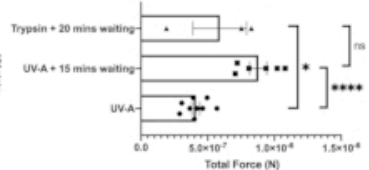

Figure 3. Local UV-A illumination TFM (LUVI-TFM) enables local traction force measurements inside a large field of view. (A) Scheme of local UV-A illumination TFM. Cells are treated with a high dose of UV-A laser to get the undeformed (reference) image. (B) The brightfield image of a single cell before UV-A laser illumination. Middle: The brightfield image of a single cell after UV-A laser illumination. Right: Traction force of a single MEF adhering to a fibronectin coated PA hydrogel $(E=5.74 \mathrm{kPa}$ ) illuminated with a $50 \mu \mathrm{m}$ diameter UV-light beam (red dashed line). (C) Left: Recording of traction forces of a cluster of mouse embryonic fibroblasts (MEF) adhering to micropatterned fibronectin (circle, $100 \mu \mathrm{m}$ diameter). The cluster was illuminated with a $200 \mu \mathrm{m}$ diameter UV-light beam (red dashed line). Right: Recording of traction forces of a cluster of mouse embryonic fibroblasts (MEF) adhering to micropatterned fibronectin (circle, $300 \mu \mathrm{m}$ diameter). The cluster was illuminated with a $300 \mu \mathrm{m}$ diameter UV-light beam (red dashed line). Scale bar $=200 \mu \mathrm{m}$. (D,E) An increase in oxidative stress in cells indicated by the increased Cy5 signal intensity after a high UV-A laser dose is detected by fluorescence microscopy. The oxidative stress leads to cell death. Scale bar $=200 \mu \mathrm{m}$. (F) Left: The stress heat map from a cluster of mouse embryonic fibroblasts (MEF) adhering to micropatterned fibronectin (circle, $100 \mu \mathrm{m}$ diameter). Right: The stress heat map from a cluster of mouse embryonic fibroblasts (MEF) adhering to micropatterned fibronectin (circle, $300 \mu \mathrm{m}$ diameter). (G) MEF cells adhering to $100 \mu \mathrm{m}$ fibronectin micropatterns slowly release their tractions after UV-A exposure. Full release is achieved after $15 \mathrm{~min}$ (the reference image was then taken $15 \mathrm{~min}$ post exposure). (H) A comparison with the conventional trypsin based-TFM for MEF cells adhering to $300 \mu \mathrm{m}$ diameter patterned-fibronectin. The result of UV-A illumination $(6,000$ $\mathrm{mJ} / \mathrm{mm}^{2}$ ) followed by $15 \mathrm{~min}$ waiting is non-significantly different from the conventional trypsin treatment (i.e., $0.05 \%$ for 20 min). The bars show mean S.E.M. (two-tailed Student's $t$-test, ${ }^{* * *} P<0.0001,{ }^{*} P<0.05$, ns $P \leq 0.5$ ). Please click here to view a larger version of this figure. 


\begin{tabular}{|c|c|c|c|c|c|}
\hline Acrylamide (\%) & $\begin{array}{c}\text { Bis- } \\
\text { acrylamide (\%) }\end{array}$ & $\begin{array}{c}\text { Acrylamide } \\
\text { from } \mathbf{4 0} \% \text { stock } \\
\text { solution (ml) }\end{array}$ & $\begin{array}{c}\text { Bis-acrylamide } \\
\text { from 2 \% stock } \\
\text { solution (ml) }\end{array}$ & Water (ml) & $\begin{array}{c}\text { Young's } \\
\text { modulus (kPa) }\end{array}$ \\
\hline 4 & 0.1 & 1 & 0.5 & 8.5 & $5,74 \pm 0,53$ \\
\hline 5 & 0.15 & 1.25 & 0.75 & 8 & $9,69 \pm 0,68$ \\
\hline 5 & 0.3 & 1.25 & 1.5 & 7.25 & $11,33 \pm 1,06$ \\
\hline
\end{tabular}

Table 1: Hydrogel stock solution mixtures and resulting elasticity

All data are deposited in the Open Access Data Repository of The Max Planck Society (Edmond) and can be accessed through the following address: https://edmond.mpdl.mpg.de/ imeji/collection/JTu8PIWqpbymN9Qf

\section{Discussion}

In this protocol, we describe the preparation of micropatterned PA hydrogels containing fluorescent beads, which are used as fiducial markers for TFM studies. Our approach is based on three steps: 1) preparation of dual-layered PA hydrogels; 2) micropatterning of ECM proteins and their transfer onto the hydrogel surface; 3) use of patterned near-UV light for TFM. The experimental setup to analyze cell tractions to the substrate requires the use of linear elastic materials with known stiffness values to calculate the forces related to displacement of the fluorescent beads ${ }^{26}$. PA hydrogels are easy to prepare, the stiffness can be easily tuned, and they are commonly used for rigidity sensing and TFM ${ }^{18,28}$. However, to obtain reproducible polymerization times and homogeneous polymerization of the entire hydrogel, attention should be paid to storing conditions and time for the reagents, e.g., APS should be kept in a desiccator to avoid losing its activity; TEMED should be protected from direct light. The use of oxidized HEA allows the covalent binding of matrix proteins on the hydrogel surface, which might be advantageous to achieve the formation of a full and stable protein layer. The oxidized HEA solution should be prepared fresh every time PA hydrogels are fabricated. The dual-layered PA hydrogel offers three main advantages: 1) it provides an alternative way to reproducibly get a homogenous distribution of fiducial beads near the hydrogel surface, without the need for making the gel extremely thin (i.e., $<20 \mu \mathrm{m}$ ). Control over hydrogel thickness is crucial for obtaining accurate measurements with TFM. When the elastic substrate is too thin, strong adherent cells such as fibroblasts may sense and mechanically respond to the underlying rigid glass substrate 29,30 . Thick hydrogels make the image acquisition for the force reconstruction more challenging. Moreover, many microscopes will not have sufficient space to accommodate them, considering the additional thickness of the glass substrate used to attach the hydrogel, unless ultrathin microscopy slides are used. 2) In the dual-layered PA hydrogel, the homogenous distribution of fiducial beads near the surface of the PA hydrogel is achieved without using a centrifuge, but rather with a simple incubation of precise amounts of hydrogel solutions and fluorescent beads. High bead density is of significant advantage when performing the PIV analysis because it increases the resolution of the traction forces and the signal-to-noise ratio without the need for confocal microscopy. 3) Confining beads in a thin layer 
close to the cell-material interface enables imaging of traction forces with epifluorescence microscopes as well as confocal microscopes. When preparing the hydrogel, the user should make sure that it firmly adheres to the bottom glass before proceeding with the subsequent steps of the protocol. We recommend following the incubation time indicated for the polymerization of the hydrogel layers, as it might be difficult to remove the glass on top of the surface without damaging the hydrogel surface.

The most well-known techniques to measure elastic properties are AFM, nanoindentation, tensile tests, and rheometry. However, nanoindentation induces very high strains on the materials that can affect the determination of elastic properties. Tensile tests and rheometry on the other hand are macroscopic measurement techniques, whereas cells interact on a microscopic scale s1,32 $^{3}$ AFM allows measurements at the microscale with reduced strains under physiological conditions. The reliability of AFM measurements can be adversely affected if experimental details are missing (e.g., indentation force and speed) or insufficient data is recorded ${ }^{27}$. Huth et al. describes an algorithm to extract Young's moduli from AFM data, which emphasizes keeping measurement details constant ${ }^{27}$. This algorithm offers a precise and reliable determination of Young's moduli and was used for our experiments. Additionally, we measured many curves on samples fabricated on different days and gained very similar results (variation of mean values of ca. 1-2 kPa). This shows that the stiffness of our gels can be reliably predicted.

In this protocol, we use a photopatterning module to create micropatterned regions on glass, which are then transferred to the hydrogel surfaces. The micropatterning shown in this protocol is based on DMD (digital micro-mirror device)- based maskless near-UV lithography $(\lambda=375 \mathrm{~nm})^{7}$. A DMD consists of a large number of micromirrors on a chip. A single pixel corresponds to a single micro-mirror. The pixelated pattern image file from a computer is projected through DMD and focused on the surface using an objective lens. For micropatterning of proteins, the focused laser light is used to cleave repellent polymer brushes with the help of a photoinitiator. Afterwards, the exposed regions are filled with ECM proteins. This maskless ablation method offers great flexibility in designing new patterns, as it does not rely on the use of a photomask. Designing and applying a pattern is very easy as it only takes several minutes using a freeware like Inkscape. However, the number of patterns and sample produced in a short amount of time is a major drawback, as this method can only be used to pattern a single substrate each time. The photopatterning module uses a near UV solid-state laser source that emits several milliwatts. The unshielded laser beam is dangerous to the eyes and skin. Reflected and scattered light and radiation can also be dangerous. Handling must be accompanied by a safety instruction from laser officers. The most critical step in the protocol when using a photopatterning module is to ensure that during the micropatterning and the ablation the laser is properly focused on the surface. A consistent illumination dose (intensity multiplied by time) of UV-light during patterning is dependent on how the laser is focused on the surface. A weak intensity on the surface due to poor focus may result in the failure of ECM transfer to the hydrogel surface resulting in no cells becoming attached to the hydrogel.

In TFM experiments, after the initial imaging of adherent cells and the fiducial markers, cells are released from the PA hydrogel by trypsin treatment to record their relaxed state. A drawback in performing this step is handling the sample 
on the microscopy stage. Without a perfusion chamber, opening the lid of the dish, aspirating the medium, rinsing, and pipetting trypsin solution represent a challenge for beginners and experienced users. In fact, these procedures are a major source of the drift in $x y z$ axes, resulting in loss of position and focus. Our local-UV illumination protocol makes TFM a more accessible technique for beginners. It should be noted that we used a commercially available microscopy-based maskless photopatterning module, but in principle any UVA lighting system could be used, eventually in combination with a mask to protect regions of the substrates, where cell traction forces should not be recorded. Exposing cells with a significant dose of low wavelength visible light (e.g., the violet light that excites DAPI emission) will lead to increasing oxidative stress, which can result in phototoxicity and cell death. Therefore, this technique can be applied even in an epifluorescence microscope without a UV laser module. However, as the intensity is much weaker, it will be much easier to accomplish TFM with the enzymatic treatment in this case.

With LUVI-TFM it is possible to use the same sample for several measurements because of the local detachment of single cells or small groups of cells. However, attention should be paid to the selection of cells to detach, to avoid recording forces from neighboring cells. Thus, for single cell measurements in absence of micropatterns, crowded regions should be avoided; for measurements on single micropatterned cells, the patterns should be designed such that the distance between single structures is at least twice the diameter of the patterned area. We also recommend not using cells from adjacent patterns in sequence, but rather sampling them from distant regions on the substrate. Our measurement is well conducted on an epifluorescence microscope equipped with a focus control feature and a 40

$x$ air NA $=0.9$ lens, where the working distance of the lens is sufficiently long and the rapid movement from one well to another is highly tunable. The targeted detachment of cells for TFM applications is effective for measuring cellular force of a single cell or of an entire small cell cluster (e.g., up to $300 \mu \mathrm{m}$ in diameter). Using our setup, we observed cell rounding and detachment for UV treatment of single cells (Figure 3A), whereas detachment seldom occurred for small cell clusters. This might lead to an underestimation of cell traction forces. For larger cell clusters, enzymatic detachment is recommended, since the users should use a 20x air objective to image the entire cluster and a focus control feature is needed. As the depth of focus of the $20 x$ air lens is much longer, the handling will not be as critical as the higher magnification objective. The user should ensure that the focus is correctly set for the ablation of cells, since the illumination dose is dependent on the laser focus on the surface. While we are aware of the possible limitations in using LUVI-TFM in cell collectives because of possible mechanical interactions with neighboring untreated cells, this aspect could actually turn out to be useful for studies on the mechanics of targeted cell extrusion, e.g., from epithelial monolayers.

In conclusion, with our TFM approach combined with light induced release of micropatterned cells, we provide a robust and high-throughput protocol to measure cell adhesion forces. The versatility of this method could be further exploited using microscopy and imaging setup aimed at improving resolution and sensitivity.

\section{Disclosures}

The authors have nothing to disclose.

\section{Acknowledgments}


We thank Mrs. Rebecca Alvarado for the support in the protocol video production and Mr. Stephen Casale for constructive criticism on the manuscript. We thank the colleagues from the Department of Cellular Biophysics, Max Planck Institute for Medical Research for the helpful discussions. The financial support from the Max-Planck-Gesellschaft to E.A.C.-A. and the Deutsche Forschungsgemeinschaft (DFG SFB1129, Projektnummer 240245660, P15 to E.A.C.-A. and P4 to U.S.S.; DFG EXC 2082/1-390761711 to U.S.S.) is also greatly acknowledged. J.B. thanks the Carl Zeiss Foundation for financial support. E.A.C.-A., C.S. and U.S.S. acknowledge funding through the Max Planck School Matter to Life supported by the German Federal Ministry of Education and Research (BMBF). C.S. is supported by the European Research Council (Consolidator Grant PHOTOMECH, no.101001797).

\section{References}

1. Geiger, B. Bershadsky, A. Exploring the neighborhood: adhesion-coupled cell mechanosensors. Cell. 110 (2), 139-142 (2002).

2. Beningo, K. A., Hamao, K., Dembo, M., Wang, Y.-I., Hosoya, $\mathrm{H}$. Traction forces of fibroblasts are regulated by the Rho-dependent kinase but not by the myosin light chain kinase. Archives of Biochemistry and Biophysics. 456 (2), 7 (2006).

3. Rape, A. D., Guo, W. H., Wang, Y. L. The regulation of traction force in relation to cell shape and focal adhesions. Biomaterials. 32 (8), 2043-2051 (2011).

4. Kumar, R., Saha, S., Sinha, B. Cell spread area and traction forces determine myosin-II-based cortex thickness regulation. Biochimica Biophysica Acta. Molecular Cell Research. 1866 (12), 118516 (2019).
5. Chen, C. S., Mrksich, M., Huang, S., Whitesides, G. M., Ingber, D. E. Geometric control of cell life and death. Science. 276 (5317), 1425-1428 (1997).

6. Thery, M. Micropatterning as a tool to decipher cell morphogenesis and functions. Journal of Cell Science. 123 (Pt 24), 4201-4213 (2010).

7. Strale, P. O. et al. Multiprotein printing by light-induced molecular adsorption. Advanced Materials. 28 (10), 2024-2029 (2016).

8. Azioune, A., Carpi, N., Tseng, Q., Théry, M., Piel, M. in Microtubules: in vivo Methods in Cell Biology. 133-146 (2010).

9. Tseng, Q. et al. A new micropatterning method of soft substrates reveals that different tumorigenic signals can promote or reduce cell contraction levels. Lab on a Chip. 11 (13), 2231-2240 (2011).

10. Vignaud, T., Ennomani, H., Thery, M. Polyacrylamide hydrogel micropatterning. Methods Cell Biology. 120, 93-116 (2014).

11. Munevar, S., Wang, Y., Dembo, M. Traction force microscopy of migrating normal and $\mathrm{H}$-ras transformed 3T3 fibroblasts. Biophysical Journal. 80 (4), 1744-1757 (2001).

12. Style, R. W. et al. Traction force microscopy in physics and biology. Soft Matter. 10 (23), 4047-4055 (2014).

13. Polacheck, W. J., Chen, C. S. Measuring cell-generated forces: a guide to the available tools. Nature Methods. 13 (5), 415-423 (2016).

14. Schwarz, U. S., Soine, J. R. Traction force microscopy on soft elastic substrates: A guide to recent computational advances. Biochimica Biophysica Acta. 1853 (11 Pt B), 3095-3104 (2015). 
15. Roca-Cusachs, P., Conte, V., Trepat, X. Quantifying forces in cell biology. Nature Cell Biology. 19 (7), 742-751 (2017).

16. Hanke, J., Probst, D., Zemel, A., Schwarz, U. S., Koster, S. Dynamics of force generation by spreading platelets. Soft Matter. 14 (31), 6571-6581 (2018).

17. Butler, J. P., Tolic-Norrelykke, I. M., Fabry, B., Fredberg, J. J. Traction fields, moments, and strain energy that cells exert on their surroundings. American Journal of Physiology. Cell Physiology. 282 (3), C595-605 (2002).

18. Sabass, B., Gardel, M. L., Waterman, C. M., Schwarz, U. S. High resolution traction force microscopy based on experimental and computational advances. Biophysical Journal. 94 (1), 207-220 (2008).

19. Willert, C. E., Gharib, M. Digital particle image velocimetry. Experiments in Fluids. 10, 181-193 (1991).

20. Lourenco, L., Krothapalli, A. On the accuracy of velocity and corticity measurements with PIV. Experiments in Fluids. 18, 421-428 (1995).

21. Adrian, R. J., Westerweel, J. Particle Image Velocimetry. Cambridge University Press (2011).

22. Westerweel, J., Scarano, F. Universal outlier detection for PIV data. Experiments in Fluids. 39 (6), 1096-1100 (2005).

23. Dembo, M., Wang, Y. Stresses at the cell-to-substrate interface during locomotion of fibroblasts. Biophysical Journal. 76 (4), 2307-2316 (1999).

24. Schwarz, U. S. et al. Calculation of forces at focal adhesions from elastic substrate data: the effect of localized force and the need for regularization. Biophysical Journal. 83 (3), 1380-1394 (2002).
25. Huang, Y., Gompper, G., Sabass, B. A Bayesian traction force microscopy method with automated denoising in a user-friendly software package. Computer Physics Communications. 256, 107313 (2020).

26. Vedadghavami, A. et al. Manufacturing of hydrogel biomaterials with controlled mechanical properties for tissue engineering applications. Acta Biomaterialia. 62, 42-63 (2017).

27. Huth, S., Sindt, S., Selhuber-Unkel, C. Automated analysis of soft hydrogel microindentation: Impact of various indentation parameters on the measurement of Young's modulus. PLoS One. 14 (8), e0220281 (2019).

28. Oria, R. et al. Force loading explains spatial sensing of ligands by cells. Nature. 552 (7684), 219-224 (2017).

29. Buxboim, A., Rajagopal, K., Brown, A. E., Discher, D. E. How deeply cells feel: methods for thin gels. Journal of Physics. Condensed Matter. 22 (19), 194116 (2010).

30. Sen, S., Engler, A. J., Discher, D. E. Matrix strains induced by cells: Computing how far cells can feel. Cell and Molecular Bioengineering. 2 (1), 39-48 (2009).

31. Arnold, M. et al. Induction of cell polarization and migration by a gradient of nanoscale variations in adhesive ligand spacing. Nano Letters. 8 (7), 2063-2069 (2008).

32. Galluzzi, M. et al. Atomic force microscopy methodology and AFMech Suite software for nanomechanics on heterogeneous soft materials. Nat Communications. 9 (1), 3584 (2018). 